\title{
Métricas para el desarrollo de software para el control adminis- trativo de proyectos en la oficina general de extensión universi- taria y proyección social de la UNCP
}

\author{
Metrics for the development of software for the administrative control of pro- \\ jects in the general office of university extension and social projection of the \\ UNCP
}

\author{
Henry G. Maquera, (") Orlando C. Ataucusi, (") Jorge A. Vega(") y Marjorie G. del Carmen Delgado" \\ (1) Facultad de Ingeniería de Sistemas, Universidad Nacional del Centro del Perú \\ Email: henry.maquera@gmail.com
}

\section{Resumen}

Las empresas vienen utilizando herramientas de software en sus diferentes procesos en forma más cotidiana. El software utilizado debe responder a los requerimientos establecidos por la empresa cliente a fin que se pueda satisfacer las necesidades identificadas. Por ello la ingeniería de software debe garantizar un producto de calidad a medida capaz de satisfaces las necesidades de la organización. Existe entonces la necesidad de evaluar la calidad del producto de software a fin de exponer claramente el grado de impacto positivo o negativo en una organización. La aplicación de métricas del modelo de calidad de software establecido por la ISO 9126 ha permitido evidenciar el incremento del grado de las métricas en el proceso de desarrollo e impacto en los usuarios coordinadores de facultad. El incremento de la evaluación de métricas de software en un promedio de $93.46 \%$ permite identificar el grado de evolución de la calidad de software en el proceso de desarrollo. Las métricas que mayor evolución han expresado son: mantenibilidad, portabilidad y calidad de uso, entonces se infiere que no es posible desarrollar un producto de software de calidad sin la participación cooperativa entre especialistas desarrolladores y los usuarios coordinadores de facultad que son los directos impactados en la UNCP.

Palabras clave: métrica, software, calidad, evolución

\begin{abstract}
Companies are using software tools in different processes in more everyday. The software used must meet the requirements set by the client company so that it can meet the identified needs. Why software engineering should ensure a quality product capable of as you meet the needs of the organization. There is thus the need to assess the quality of the software product to clearly state the degree of positive or negative impact on an organization. The application of metrics software quality model established by ISO 9126 have spotlighted the increasing degree of metrics in the development process and impact on users coordinators faculty. The increase in software metrics evaluation at an average of $93.46 \%$ to identify the degree of evolution of quality in the software development process. The metrics further evolution expressed are: maintainability, portability and quality of use, then it follows that it is not possible to develop a software product quality without the cooperative participation among specialists developers and coordinators users faculty who are directly impacted the UNCP.
\end{abstract}

Keywords : metrics, software, quality, evolution 


\section{Introducción}

Las métricas de calidad de software son medidas cuantitativas que permiten determinar el grado de eficacia y eficiencia del proceso de desarrollo de proyectos de software que permiten identificar el grado de cumplimiento de requisitos, grado de satisfacción del producto, grado de satisfacción del cliente a través de la recopilación de datos entre los actores involucrados. Al ser el producto de software un activo intangible no existe una forma exacta de medir el software, por lo que es pertinente definir métricas internas y externas de calidad a fin de establecer ítems apropiados para determinar la calidad del software a través de la ISO 9126.

La ISO 9126 ofrece un estándar internacional para la evaluación de la calidad de un producto de software basada en métricas de funcionalidad, fiabilidad, usabilidad, eficiencia, mantenibilidad, portabilidad, calidad de uso y permite establecer datos estadísticos a fin exponer un escenario real del impacto de la solución de software en la Oficina General de Extensión Universitaria y Proyección Social.

\section{Problema}

¿De qué manera evaluar el desarrollo de software de administración de proyectos de la Oficina General de Extensión Universitaria y Proyección Social de la UNCP?

\section{Objetivo}

Evaluar el desarrollo del software de administración de proyectos de la Oficina General de Extensión Universitaria y Proyección Social de la UNCP mediante el modelo de calidad de la norma ISO 9126

\section{Materiales y métodos}

La metodología que ha utilizado para la realización del trabajo de investigación se ha basado en la aplicación de métricas establecidas por el modelo de calidad de la ISO 9126.

Tabla I. Métricas del modelo de calidad de software

\begin{tabular}{ll} 
Mútrica & \multicolumn{1}{c}{ Descripción } \\
Funcionalidad & Grado de satisfacción de las \\
& funciones implícitas y explicitas \\
& requeridas en el software. \\
& $-\quad$ Adecuación. \\
& $-\quad$ Exactitud. \\
& $-\quad$ Interpolaridad. \\
& $-\quad$ Seguridad. \\
Fiabilidad & Grado en que el software \\
& mantiene su nivel adecuado de \\
& operación en las condiciones \\
& establecidas. \\
& $-\quad$ Madurez. \\
& $-\quad$ Capacidad de recuperación. \\
& $-\quad$ Tolerancia a fallos. \\
& $-\quad$ Cumplimiento de fiabilidad.
\end{tabular}

\begin{tabular}{|c|c|}
\hline Usabilidad & $\begin{array}{l}\text { Grado de facilidad de operar el } \\
\text { software y de ser entendible para } \\
\text { el usuario final. } \\
\text { - } \quad \text { Aprendizaje. } \\
\text { - } \quad \text { Comprensión. } \\
\text { - } \quad \text { Operatividad. } \\
\text { - } \quad \text { Cumplimiento de la } \\
\quad \text { usabilidad. }\end{array}$ \\
\hline Eficiencia & $\begin{array}{l}\text { Grado de uso de recursos de } \\
\text { forma adecuada por el software } \\
\text { conservando buen desempeño y } \\
\text { sin exceso. } \\
\text { - } \quad \text { Comportamiento en el } \\
\text { tiempo } \\
\text { - Comportamiento de recursos } \\
\text { - } \quad \text { Cumplimiento de eficiencia. }\end{array}$ \\
\hline Mantenibilidad & $\begin{array}{l}\text { Grado de facilidad en modificar el } \\
\text { código fuente o agregar funciones } \\
\text { para responder a los nuevos } \\
\text { requerimientos } \\
\text { - } \quad \text { Estabilidad. } \\
\text { - } \quad \text { Facilidad de análisis. } \\
\text { - } \quad \text { Facilidad de cambio. } \\
\text { - } \quad \text { Facilidad de pruebas. } \\
\text { - } \quad \text { Cumplimiento de } \\
\quad \text { mantenibilidad. }\end{array}$ \\
\hline Portabilidad & $\begin{array}{l}\text { Grado de movilidad del sistema o } \\
\text { uno de sus componentes de una } \\
\text { plataforma a otra sin mayor } \\
\text { esfuerzo } \\
\text { - } \quad \text { Capacidad de instalación. } \\
\text { - } \quad \text { Capacidad de reemplazar. } \\
\text { - } \quad \text { Coexistencia. } \\
\text { - } \quad \text { Adaptabilidad. } \\
\text { - } \quad \text { Cumplimiento de la } \\
\quad \text { portabilidad. }\end{array}$ \\
\hline $\begin{array}{l}\text { Calidad de } \\
\text { uso }\end{array}$ & $\begin{array}{l}\text { Grado de seguridad y } \\
\text { confiabilidad del usuario con el } \\
\text { uso del software } \\
\text { - } \quad \text { Eficacia. } \\
\text { - } \quad \text { Productividad. } \\
\text { - } \quad \text { Seguridad. } \\
\text { - } \quad \text { Satisfacción. }\end{array}$ \\
\hline
\end{tabular}

Adicionalmente se utilizó la siguiente tabla de criterio de valoración numérica para cada métrica establecida por el modelo de calidad de la ISO 9126.

Tabla I. Métricas del modelo de calidad de software

\begin{tabular}{|c|c|}
\hline Valor & Criterio \\
\hline 10 & $\begin{array}{l}\text { Extremo cambio en el grado en la } \\
\text { métrica }\end{array}$ \\
\hline $\begin{array}{l}9 \\
8\end{array}$ & Muy alto cambio en el grado en la métrica \\
\hline $\begin{array}{l}7 \\
6\end{array}$ & Alto cambio en el grado en la métrica \\
\hline 5 & Cambio medio en el grado en la métrica \\
\hline $\begin{array}{l}4 \\
3\end{array}$ & Bajo cambio en el grado en la métrica \\
\hline $\begin{array}{l}2 \\
1\end{array}$ & $\begin{array}{l}\text { Muy bajo cambio en el grado en la } \\
\text { métrica }\end{array}$ \\
\hline 0 & $\begin{array}{l}\text { Irrelevante a cambio en el grado en la } \\
\text { métrica }\end{array}$ \\
\hline
\end{tabular}

\section{Resultados}

El desarrollo de la solución de software se ha llevado a cabo mediante la metodología de desarrollo 
de software denominada Programación Xtrema. Al inicio de esta investigación no se tenía software alguno que sirva de punto de inicio para un análisis del incremento en el grado de las métricas definidas. Como población y muestra de investigación se contó con la participación de todos los coordinadores de extensión universitaria y proyección social de las diversas facultades de la Universidad Nacional del Centro del Perú.

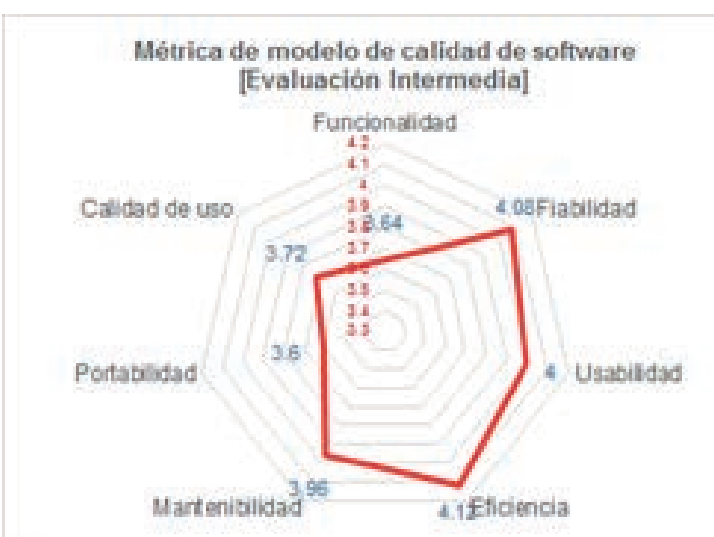

Figura I.

I proceso de evaluación de las métricas fue considerado en tres hitos. La primera evaluación fue llevada a cabo al inicio del proceso de desarrollo, donde se tuvo los grados obtenidos por cada métrica del modelo de calidad fue 0. La evaluación intermedia presentó los resultados mostrados en la figura I. La evaluación final por cada métrica del modelo de software se presenta en la figura 2.

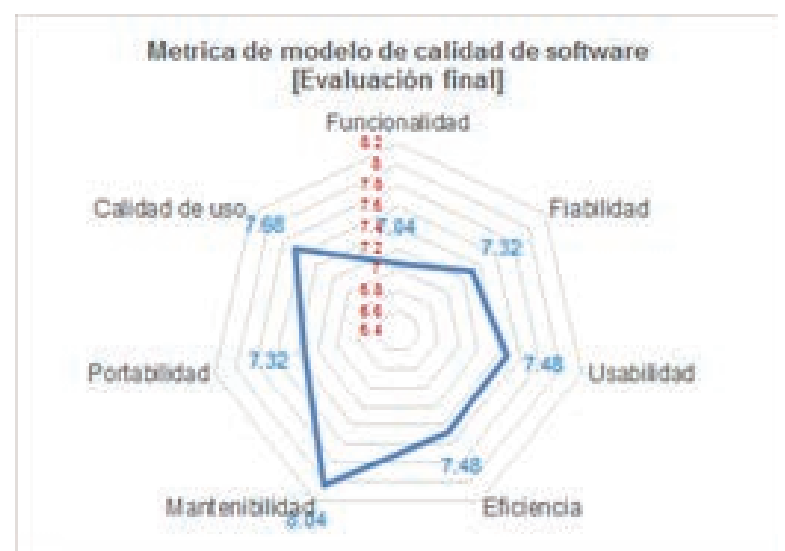

Figura 2.

La asignación de valores para cada métrica por cada coordinador de extensión universitaria y proyección social bajo la guía y explicación de los miembros responsables de la investigación. Para cada métrica de software se consolido el promedio a fin de establecer una información resumida.

Al realizar una comparación de los resultados obtenidos se obtuvo la figura 3 que expone el incremento de los grados de evolución de cada métrica del modelo de calidad de software.

Métrica de modelo de calidad de software [Evolución en la evaluación]

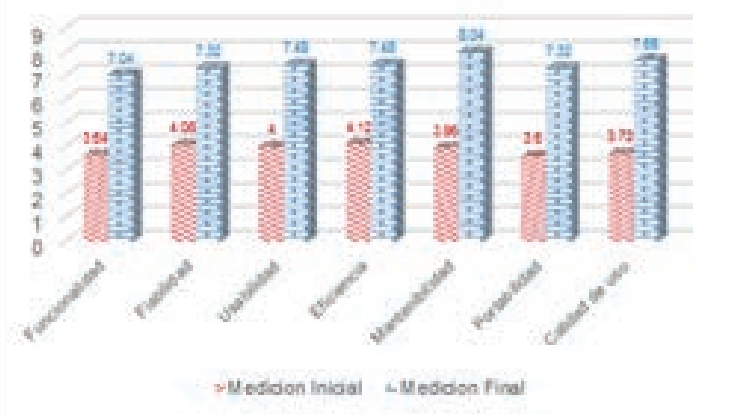

Figura 3.

\section{Discusión}

La evolución en los grados de evaluación de las métricas evidencian que el impacto del producto de software entre los coordinadores de la oficina general de extensión universitaria y proyección social. Y la aplicación de métricas del modelo de calidad de software permite determinar un análisis para cada métrica establecida:

- Métrica de funcionalidad. Esta métrica se incrementa en un $93.41 \%$. A lo largo del proceso de desarrollo del producto de software se ha contado con la participación activa de los coordinadores de facultades y del personal administrativo de la OGEUPS.

- Métrica de fiabilidad. Esta métrica se incrementa en un $79.41 \%$. Se ha sometido al software a pruebas de estrés de procesamiento concurrente y procesamiento de equipos.

- Métrica de usabilidad. Esta métrica se incrementa en un $87 \%$. Se evidencia en la capacidad de los usuarios en la rápida adaptabilidad de uso de esta solución de software.

- Métrica de eficiencia. Esta métrica se incrementa en un $81.55 \%$. Esta métrica fue evaluado por el personal de la OGl debido a su experiencia y capacitación en la evaluación de consumo de recursos en el tiempo de procesamiento de software.

- Métrica de mantebilidad. Esta métrica se incrementa en un 103.03\%. Esta métrica fue evaluado por el personal de la $\mathrm{OGl}$ debido al grado de responsabilidad en salvaguardar los códigos fuentes del producto de software.

- Métrica de Portabilidad. Esta métrica se incrementa en un 103.33\%. Esta métrica fue evaluado por el personal de la $\mathrm{OG}$ debido al grado de responsabilidad 
en la instalación del producto de software en diferentes versiones de sistemas operativos existentes entre los coordinadores de facultad.

- Métrica de calidad de uso. Esta métrica se incrementa en un $106.45 \%$. Se evidencia en los diversos niveles de aceptación de los coordinadores en la reducción significativa de recursos consumidos en el desarrollo de actividades propias del cargo de coordinador de facultad.

\section{Conclusiones}

I. Comúnmente los procesos en la oficina de extensión universitaria y proyección social de la UNCP y facultades se ha venido realizando de forma manual. Ello significaba que los procesos eran lentos y de alto riesgo en su ejecución. La aplicación de un producto de software evaluado mediante métricas de calidad de software ha permitido evidenciar el impacto positivos en las actividades asignadas a los coordinadores de extensión universitaria y proyección social de las facultades y de la oficina general.

2. La aplicación de una metodología ágil de desarrollo de software permite establecer soluciones a medida en un tiempo relativamente corto, sin embargo carecen de métricas de impacto dentro de las mismas. Por lo tanto la aplicación de métricas de calidad de software permiten lograr valores cuantitativos para lograr detectar los índices de mejora por parte de la solución tecnológica en la organización.

3. La evaluación de métricas en una etapa intermedia y su respectivo incremento en una etapa final permitieron determinar el impacto positivo en el desarrollo de la solución de software. Por lo tanto la aplicación de métricas de calidad de software ha permitido establecer estrategias coherentes en el desarrollo de un producto de software de calidad.

4. Una de las métricas de mayor incremento son: mantenibilidad, portabilidad y calidad de uso. Sin embargo los actores involucrados son el personal de la OGl y los usuarios coordinadores de facultades. Por ello se infiere que una solución de software requiere de la participación de especialistas desarrolladores capaces de permitir el crecimiento del producto y de los actores finales que reciben el impacto directo de la aplicación del producto de software.

\section{Referencias}

Campderrich Benet. Ingeniería del Software. (2003). España. Editorial Pearson Education. S.A.

Mann, Mik. Ingeniería del Software. (2006). España. Editorial McGraw Hill S.A.
Piattini, M., García F., Caballero, I. Calidad de los Sistemas Informáticos. (2007). Perú. Editorial Alfaomega.

Sommerville, lan. Ingeniería del Software. (2005). España. Séptima Edición. Editorial Pearson Educación S.A.

Whitten, Jeffrey l.; Bentley, lonnie, Dittman, kevin. (2004). Systems Analysis and Design Methods. USA. Sexta Edición, Edit. McGraw-Hill Irwin. 